\title{
Silencing glioma-associated oncogene homolog 1 suppresses the migration and invasion of hepatocellular carcinoma in vitro
}

\author{
ZEMING HU ${ }^{1,2}$, FANGFANG XIE ${ }^{2}$, ANG HU ${ }^{2}$, MENGJING XU ${ }^{2}$, YUWEN LIU ${ }^{1,2}$, JIANKANG ZHANG ${ }^{1}$, \\ JIANBO XIAO $^{1,2}$, YUNLEI SONG ${ }^{2}$, JIANING ZHONG ${ }^{2}$ and BIN CHEN $^{1}$ \\ ${ }^{1}$ Department of General Surgery, The First Affiliated Hospital of Gannan Medical University; \\ ${ }^{2}$ Key Laboratory of Prevention and Treatment of Cardiovascular and Cerebrovascular Diseases, \\ Ministry of Education, Gannan Medical University, Ganzhou, Jiangxi 341000, P.R. China
}

Received January 6, 2020; Accepted August 20, 2020

DOI: $10.3892 / \mathrm{ol} .2020 .12091$

\begin{abstract}
Hepatocellular carcinoma (HCC) is the fourth most common cause of cancer-associated death worldwide. Glioma-associated oncogene homolog 1 (Gli1) is a key component and functions as a reliable marker of Hedgehog signaling pathway activation. Previous studies have demonstrated that Glil serves important roles in the progression of various types of cancer, including HCC. However, its effect on HCC invasion and metastasis and the underlying mechanism remain to be elucidated. Small interference RNA was employed to silence the Gli1 gene in liver cancer cells. Reverse transcription-quantitative PCR and western blot analysis were performed to evaluate the mRNA and protein expression of Gli1, respectively. A series of assays, including Cell Counting Kit-8, adhesion, wound healing and Matrigel invasion were performed to investigate cell viability, adhesive, migratory and invasive capabilities of liver cancer cells, respectively. In addition, immunofluorescence staining was performed to determine the cellular localization of focal adhesion kinase (FAK), phosphorylated (p-)FAK and p-AKT. The mRNA and protein expression of Gli1 in liver cancer cells (HepG2 and SK-Hep1) were markedly decreased in a dose-dependent manner following Glil-knockdown. Gli1 silencing significantly inhibited the adhesion, migration and invasion of SK-Hep1 cells. Additionally, knockdown of Gli1 markedly suppressed the expression of metalloproteinase (MMP)-2 and
\end{abstract}

Correspondence to: Professor Jianing Zhong, Key Laboratory of Prevention and Treatment of Cardiovascular and Cerebrovascular Diseases, Ministry of Education, Gannan Medical University, 323 National Road, Ganzhou, Jiangxi 341000, P.R. China

E-mail: zhongning_003@163.com

Professor Bin Chen, Department of General Surgery, The First Affiliated Hospital of Gannan Medical University, 23 Youth Road, Ganzhou, Jiangxi 341000, P.R. China

E-mail: chenb1970829@163.com

Key words: hepatocellular carcinoma, glioma-associated oncogene homolog 1 , adhesion, migration, invasion
MMP-9. Furthermore, downregulation of Gli1 blocked the FAK/AKT signaling pathway. Gli1 serves significant roles in the migration and invasion of $\mathrm{HCC}$ cells through activation of the FAK/AKT signaling pathway and subsequent upregulation of MMP-2 and MMP-9 expression. Thus, Gli1 may be a potential protein target for the regulation of HCC migration and invasion.

\section{Introduction}

Hepatocellular carcinoma (HCC) is the fourth most common cause of cancer-associated death worldwide $(1,2)$. Due to the limited improvements in the diagnosis and treatment of HCC over the past two decades, the prognosis and survival rate of patients with HCC remain poor $(3,4)$. The high mortality rate of HCC, with $\sim 782,000$ deaths annually, is largely caused by its metastases and recurrence (5). Therefore, an understanding of the mechanisms underlying the invasion and metastasis of $\mathrm{HCC}$ is key to improving the treatment of this disease.

Previous studies have demonstrated that aberrant activation of the Hedgehog ( $\mathrm{Hh}$ ) signaling pathway serves a critical role in the tumorigenesis and progression of various types of cancer, including pancreatic, breast, lung, ovarian, gastrointestinal cancer and HCC (6-8). As a key component of the Hh signaling pathway, glioma-associated oncogene homolog 1 (Gli1) is a member of the family of zinc finger transcription factors that functions as a downstream protein in the Hh signaling pathway. Gli1 is a reliable activation marker of the $\mathrm{Hh}$ signaling pathway (9). Emerging evidence has demonstrated that Gli1 is overexpressed in a number of types of cancer, including ovarian (10), prostate (11), gastric (12), esophageal squamous cell (13), pancreatic (14) and liver cancer (15). A recent study concluded that hyperactivation of Glil is sufficient to trigger the uncontrolled progression of cancer features such as proliferation, migration and invasion (16). However, the underlying mechanisms of Gli1 in the invasion and metastasis of HCC remain to be elucidated.

The focal adhesion kinase-phosphoinositide 3-kinase-AKT (FAK/PI3K/AKT) axis is a critical pathway that regulates the invasion and metastasis of HCC (17). Briefly, FAK, a non-receptor protein tyrosine kinase, is an essential factor that regulates the PI3K/AKT signaling 
pathway, which is associated with a poor prognosis in different types of cancer (18). Activation of FAK can phosphorylate PI3K and subsequently lead to the phosphorylation of AKT (19). Phosphorylation of FAK is important for invasion and metastasis as it regulates the expression of matrix metalloproteinase (MMP)-2 and MMP-9 in HCC (20). Overexpression of FAK has been detected in various malignant tumors, including breast, prostate, colorectal and ovarian cancer (21-24). Ectopic expression of FAK and phosphorylated (p-)FAK are associated with the invasion and metastasis of HCC $(20,25)$. Furthermore, phosphorylation of FAK can activate the PI3K/AKT signaling pathway, leading to regulation downstream of the pathway that transduces a $\beta 1$ integrin viability signal in collagen matrices (26).

The present study evaluated the roles of Gli1 in liver cancer cells. Gaining an understanding of the mechanism underlying the function of Gli1 in the invasion and metastasis of HCC will provide a potential target to control the invasion and metastasis of HCC.

\section{Materials and methods}

Cell lines and culture. Human liver cancer cell lines (HepG2 and SK-Hep1) were purchased from the Cell Bank of the Chinese Academy of Sciences. Cells were cultured in RPMI-1640 (Biological Industries) supplemented with 10\% fetal bovine serum (FBS; Biochrom, Ltd.) and 1\% penicillin/streptomycin (Invitrogen; Thermo Fisher Scientific, Inc.) in a humidified $5 \% \mathrm{CO}_{2}$ atmosphere at $37^{\circ} \mathrm{C}$.

RNA interference. Small interfering (si)RNA specific for Gli1 (si-Gli1) sequences and scrambled negative control (NC) siRNA with Glil analogue nonsense sequences were obtained from Sangon Biotech Co., Ltd. and were as follows: Gli1 (sense: 5'-CCAGUGUCCUCGACUUGAAdTdT-3'; antisense: 5'-UUC AAGUCGAGGACACUGGTdTd-3') and NC (sense: 5'-UUC UCCGAACGUGUCACGUTT-3'; antisense: 5'-ACGUGACAC GUUCGGAGAATT-3'). Cells were seeded into 6-well plates and cultured to $80 \%$ confluence and then transfected with $50 \mathrm{nmol} / 1 \mathrm{NC}$ siRNA, and 50 or $100 \mathrm{nmol} / \mathrm{l} \mathrm{si}-\mathrm{Gli} 1$ for $24 \mathrm{~h}$ at $37^{\circ} \mathrm{C}$ using Lipofectamine ${ }^{\circledR} 3000$ (Invitrogen; Thermo Fisher Scientific, Inc.) according to the manufacturer's instructions. Briefly, cells were divided into four groups: A blank control (CTRL) group (only treated with serum-free medium), a NC group (transfected with NC siRNA), a $50 \mathrm{nmol} / \mathrm{l}$ si-Gli1 group and a $100 \mathrm{nmol} / \mathrm{l}$ si-Gli1 group. All subsequent experiments were performed $24 \mathrm{~h}$ post-transfection.

Reverse transcription-quantitative $(R T-q) P C R$. Total RNA was extracted from liver cancer cells (HepG2 and SK-Hep1) using TRIzol ${ }^{\circledR}$ reagent (Invitrogen; Thermo Fisher Scientific, Inc.) and $5 \mu \mathrm{g}$ RNA was reverse transcribed to complementary DNA (cDNA) using a RevertAid First Strand cDNA synthesis kit (Invitrogen; Thermo Fisher Scientific, Inc.). Subsequently, qPCR was performed using FastStart Universal SYBR-Green Master (cat. no. 31168620; Roche Diagnostics $\mathrm{GmbH}$ ), according to the manufacturer's protocol, and performed on an ABI 7500 Real-time PCR system (Thermo Fisher Scientific, Inc.). The thermocycling conditions were: $95^{\circ} \mathrm{C}$ Denaturation for $15 \mathrm{~min}$ and then 40 cycles of $95^{\circ} \mathrm{C}$ for
$10 \mathrm{sec}$ and $60^{\circ} \mathrm{C}$ for $30 \mathrm{sec}$. The relative mRNA expression levels of target genes were normalized to $\beta$-actin and calculated by use of the $2^{-\Delta \Delta \mathrm{Cq}}$ method (27). The specific sequences of primers were as follows: Gli1 forward: 5'-AGGGCTGCA GTAAAGCCTTCA-3', Glil reverse: 5'-CCTGACATGTTT TCGCAGCG-3'; MMP-2 forward: 5'-GACAACGCCCCC ATACCAG-3', MMP-2 reverse: 5'-CACTCGCCCCGTGTG TTAGT-3'; MMP-9 forward: 5'-ACGCAGACATCGTCATCC AGT-3', MMP-9 reverse: 5'-GGACCACAACTCGTCATC GTC-3'; $\beta$-actin forward: 5'-GTGGACATCCGCAAAGAC-3', $\beta$-actin reverse: 5'-GAAAGGGTGTAACGCAACT-3'. All experiments were performed in triplicate.

Western blotting. Briefly, protein was extracted from cells (5x106/well) using 6X sample buffer (1 M Tris-HCl, $\mathrm{pH} 6.8$, $10 \%$ SDS, $20 \%$ glycerol, $0.5 \%$ Bromphenol Blue and 2-mercaptoethanol) for $10 \mathrm{~min}$ at $100^{\circ} \mathrm{C}$ and then centrifuged at $10,000 \mathrm{x}$ g for $15 \mathrm{~min}$ on ice. The total protein concentrations were measured using a BCA protein assay kit (Thermo Fisher Scientific, Inc.) according to the manufacturer's instructions. The protein samples (10 $\mu \mathrm{g} /$ lane) were separated by $10 \%$ SDS-PAGE and transferred to polyvinylidene fluoride membranes (EMD Millipore). Subsequently, the membranes were incubated with blocking buffer (5\% non-fat dry milk and $0.05 \%$ Tween-20 in PBS) for $1 \mathrm{~h}$ at $37^{\circ} \mathrm{C}$ and the following primary antibodies overnight at $4^{\circ} \mathrm{C}$ : Rabbit anti-Gli1 (Cell Signaling Technology, Inc.; cat. no. 3538S; 1:1,000 dilution), rabbit anti-MMP-2 (Cell Signaling Technology, Inc.; cat. no. 40994S; 1:1,000 dilution), rabbit anti-MMP-9 (Abcam; cat. no. ab38898; 1:1,000 dilution), rabbit anti-FAK (Abcam; cat. no. ab40794; 1:1,000 dilution), rabbit anti-p-FAK (Abcam; cat. no. ab39967; 1:1,000 dilution), rabbit anti-PI3K (Cell Signaling Technology, Inc.; cat. no. 4257S; 1:1,000 dilution), rabbit anti-p-PI3K (Cell Signaling Technology, Inc.; cat. no. 4228S; 1:1,000 dilution), mouse anti-AKT (Cell Signaling Technology, Inc.; cat. no. 2920S; 1:1,000 dilution), rabbit anti-p-AKT (Abcam; cat. no. ab81283; 1:1,000 dilution) and mouse anti- $\beta$-Actin (Abcam; cat. no. ab8226; 1:2,000 dilution). Finally, the membranes were incubated with goat anti-rabbit or goat anti-mouse horseradish peroxidase-conjugated secondary antibodies (Sigma-Aldrich; Merck KGaA; cat. nos. A8919 and AP186P; 1:10,000 dilution) for $1 \mathrm{~h}$ at room temperature. The immunoblot bands were visualized using an enhanced chemiluminescence kit (cat. no. E1328; Beijing Lilye Science \& Technology Co., Ltd., http://www.micro-helix.com) and exposed on the gel imaging analysis system (Amersham Imager 600; Cytiva). The intensity of bands was quantified using ImageJ software (version 1.48; National Institutes of Health).

Immunofluorescence staining. The cells transfected with or without si-Gli1 were fixed in $4 \%$ paraformaldehyde/PBS for $15 \mathrm{~min}$ at $-20^{\circ} \mathrm{C}$ and then washed three times using PBS containing $0.1 \%$ Triton $\mathrm{X}-100$ (PBST) for $15 \mathrm{~min}$ at room temperature. Following blocking in PBS containing 5\% BSA for $30 \mathrm{~min}$ at $37^{\circ} \mathrm{C}$, cells were incubated with the primary antibodies rabbit anti-FAK (Abcam; cat. no. ab40794; 1:1,000 dilution), rabbit anti-p-FAK (Abcam; cat. no. ab39967; 1:1,000 dilution) and rabbit anti-p-AKT (Abcam; cat. no. ab81283; 1:1,000 dilution) for $1 \mathrm{~h}$ at room temperature. The labeled 
cells were washed three times with PBST and then incubated with Alexa Fluor 594 (goat anti-rabbit IgG; Abcam, cat. no. ab150088; 1:1,000 dilution) for $1 \mathrm{~h}$ at room temperature in the dark. After washing three times with PBST, the cell nuclei were stained with DAPI ( $1 \mu \mathrm{g} / \mathrm{ml}$, Abcam, cat. no. ab228549) for $10 \mathrm{~min}$ at room temperature in the dark and detected with a confocal laser scanning microscope (LSM 880; Zeiss AG) at x63 magnification. Cell clusters were counted in five randomly selected fields using a fluorescence microscope and the mean intensity was calculated using ImageJ software.

Cell viability assay. Cell viability was determined by Cell Counting Kit (CCK)-8 assay (Beijing Solarbio Science \& Technology Co., Ltd.; cat. no. CA-1210-500) following the manufacturer's instructions. Briefly, liver cancer cells with or without transfection of si-Gli1 were incubated in 96-plates for $24 \mathrm{~h}$ at $37^{\circ} \mathrm{C}$. Then, cells were added to $10 \mu \mathrm{l} \mathrm{CCK}-8$ staining reagent and then incubated for another $4 \mathrm{~h}$ at $37^{\circ} \mathrm{C}$. Absorbance at $450 \mathrm{~nm}$ was measured using a microplate reader (Varioskan Lux; Thermo Fisher Scientific, Inc.).

Adhesion assay. Cell adhesive abilities of SK-Hep1 cells in different groups were determined using an adhesion assay. In brief, $50 \mu$ l Matrigel (BD Biosciences; cat. no. 8036008) was added to each well in a 96 -well plate for $30 \mathrm{~min}$ at $37^{\circ} \mathrm{C}$ and then $5 \times 10^{4}$ cells were seeded in the coated plates for incubation for $4 \mathrm{~h}$ at $37^{\circ} \mathrm{C}$. Finally, cells were washed with PBS to wash away the unattached cells. The attached cells were analyzed using the CCK- 8 assay as described above.

Wound healing assay. Cell migratory abilities were detected using wound healing assay. The prepared cells treated with or without si-Gli1 were cultured in 6-well plates to $\sim 90 \%$ confluence using serum-free medium. The confluent monolayer cells were scratched gently with a $100 \mu \mathrm{l}$ pipette tip and images captured using an inverted microscope (DMi8; Leica Microsystems $\mathrm{GmbH}$; magnification, x100) at 0 and $24 \mathrm{~h}$. The wound area was quantified using ImageJ software.

Transwell invasion assay. Cell invasion assay was performed using 24-well Transwell plates with $8-\mu \mathrm{m}$ pore filters (Corning Life Sciences; cat. no. 3422) precoated with Matrigel Basement Membrane Matrix (BD Biosciences) at $37^{\circ} \mathrm{C}$ for 1-4 $\mathrm{h}$ according to the manufacturer's protocols. Briefly, $1 \times 10^{5}$ cells were suspended in $100 \mu 1$ of serum-free medium and added to the upper chamber and then $600 \mu$ l RPMI-1640 supplemented with $20 \%$ FBS was loaded into the lower chamber. After $24 \mathrm{~h}$ of incubation, the non-invaded cells in the top chamber were removed with a cotton swab and the cells fixed with $100 \%$ methanol for $20 \mathrm{~min}$ at room temperature and stained with $0.1 \%$ crystal violet (Beijing Solarbio Science \& Technology Co., Ltd.; cat. no. G1063) for $30 \mathrm{~min}$ at room temperature. The stained cells were imaged under an inverted light microscope (DMi8; Leica Microsystems $\mathrm{GmbH}$; magnification, x100) and analyzed using ImageJ software.

Statistical analysis. Statistical analysis was performed using GraphPad Prism software (version 7.0; GraphPad Software, Inc.). All experiments were performed in triplicate and data are presented as the mean \pm standard deviation. Unpaired

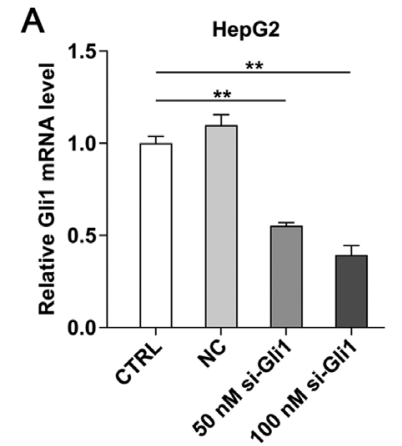

C
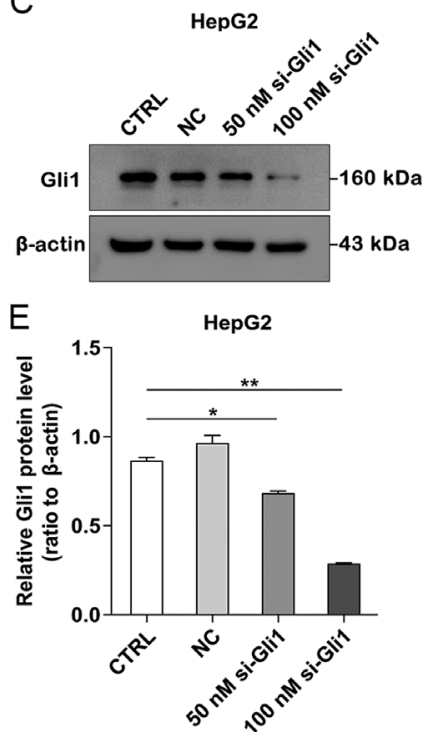

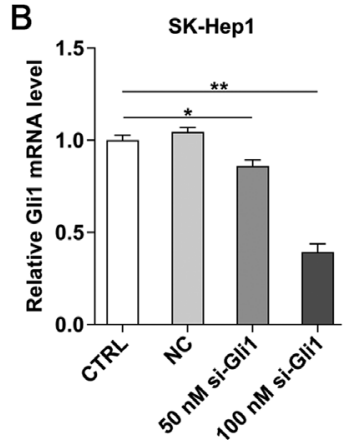

D

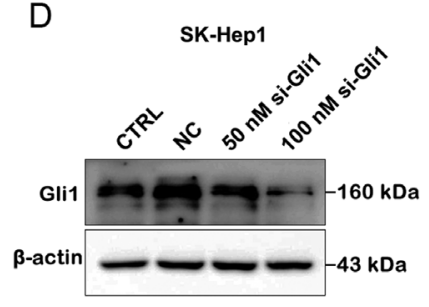

$\mathrm{F}$

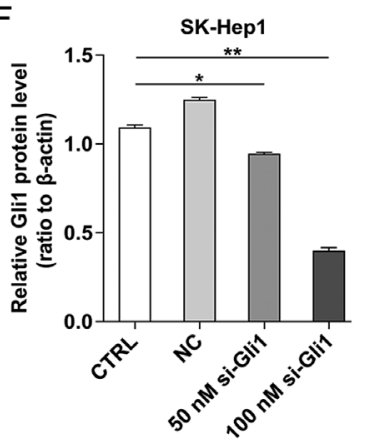

Figure 1. Downregulation of Gli1 expression in liver cancer cells by siRNA. The relative mRNA expression of Gli1 was significantly decreased in (A) HepG2 and (B) SK-Hep1 cells using specific siRNA against Gli1. The protein expression of Gli1 was markedly reduced in (C) HepG2 and (D) SK-Hep1 cells by Gli1 siRNA. (E and F) Quantification analysis of representative western blot images using ImageJ software. $\beta$-actin was used as a loading control. ${ }^{*} \mathrm{P}<0.05$ and ${ }^{* *} \mathrm{P}<0.01$ vs. CTRL group. Gill, glioma-associated oncogene homolog 1; si, small interference; CTRL, blank control group; $\mathrm{NC}$, negative control siRNA group.
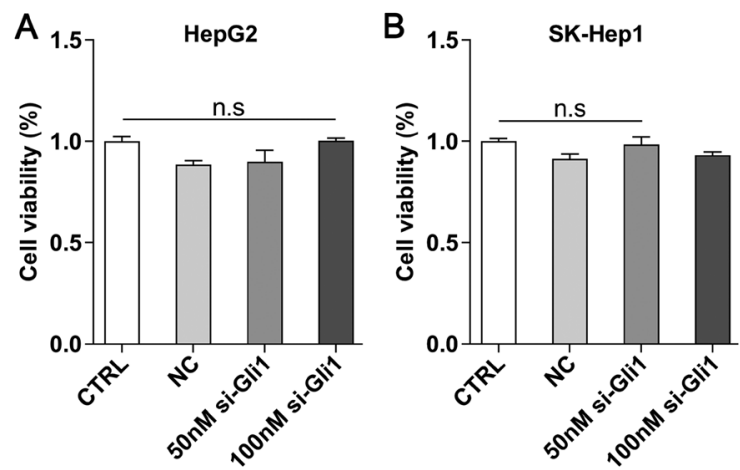

Figure 2. Gli1 siRNA had no significant effect on the viability of liver cancer cells. Cytotoxicity was detected using the Cell Counting Kit- 8 assay using (A) HepG2 and (B) SK-Hep1 cells after transfection of NC or Gli1 siRNA. Gill, glioma-associated oncogene homolog 1; si, small interference; n.s, no significant difference; CTRL, blank control group; NC, negative control siRNA group.

Student's t-test was used to compare differences between two groups, whilst one-way analysis of variance followed by 
A

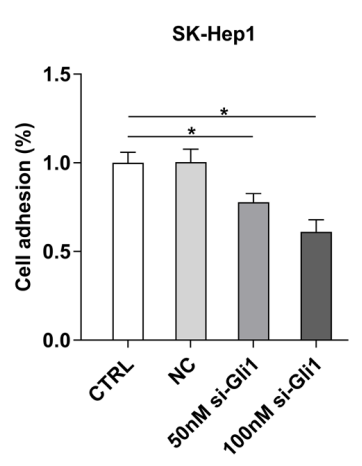

C

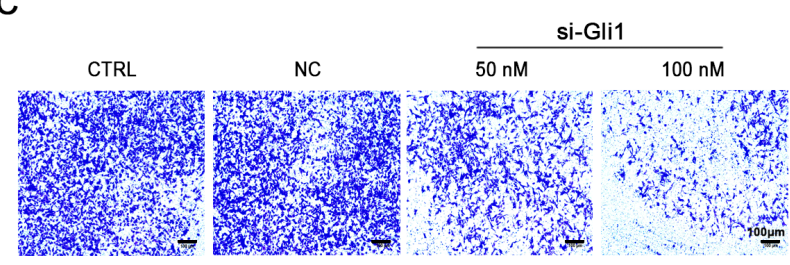

E

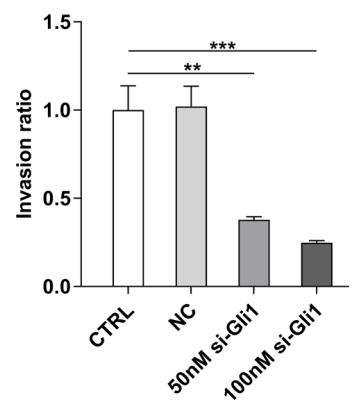

B
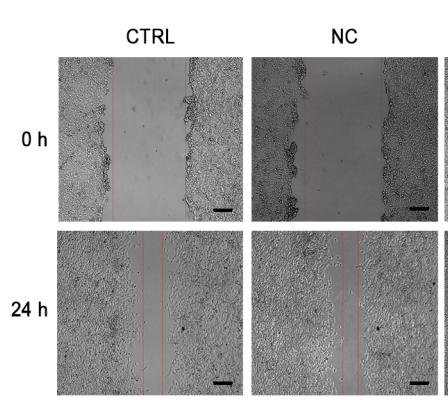

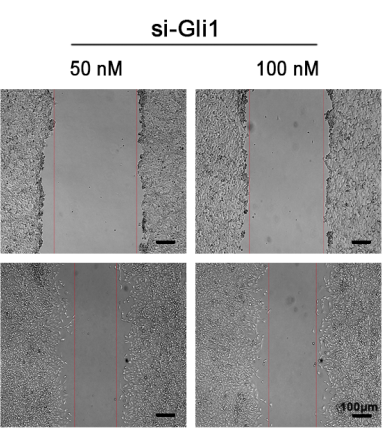

D

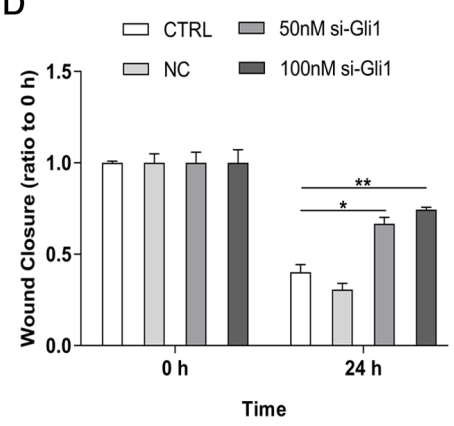

Figure 3. Gli1 interference inhibits cell adhesion, migration and invasion in SK-Hepl cells. (A) Cell adhesive ability of hepatoma cells was significantly suppressed in the si-Gli1 groups compared with the CTRL group using cell adhesion assay. (B and C) Images and (D and E) histograms showing that Gli1 siRNA markedly decreased the migration and invasion abilities of SK-Hep1 cells as demonstrated by the wound healing and Matrigel invasion assays, respectively. Scale bar, $100 \mu \mathrm{m} .{ }^{*} \mathrm{P}<0.05,{ }^{* *} \mathrm{P}<0.01$ and ${ }^{* * * *} \mathrm{P}<0.001$ vs. CTRL group. Gill, glioma-associated oncogene homolog 1 ; si, small interference; CTRL, blank control group; NC, negative control siRNA group.

Dunnett's post-hoc test were performed to compare differences between multiple groups. $\mathrm{P}<0.05$ was considered to indicate a statistically significant difference.

\section{Results}

Knockdown of Gli1 inhibits Glil expression in HepG2 and SK-Hepl cells. To understand the potential function of Gli1 in liver cancer cells, Gli1 expression was downregulated by RNA interference. The knockdown efficiency of Gli1 in liver cancer cells was determined using RT-qPCR and western blotting. The results demonstrated that the mRNA and protein expression levels were significantly decreased in a dose-dependent manner and the most efficient concentration for Glil silencing was $100 \mathrm{nmol} / \mathrm{l}$ in both HepG2 and SK-Hep1 cells $(\mathrm{P}<0.05$ and $\mathrm{P}<0.01$; Fig. 1).

To investigate whether the efficiency of Gli1 transfected with siRNA was dependent on its cellular cytotoxicity, CCK-8 assay was performed to examine the viability of HepG2 and
SK-Hep1 cells. The results demonstrated that the 50 - and $100-\mathrm{nmol} / \mathrm{l}$ si-Gli1 groups were not significantly different compared with the CTRL group ( $P>0.05$; Fig. 2$)$, which indicated that the effect of Gli1 transfection and the following experiments using liver cancer cells was independent of its cellular cytotoxicity.

Reduced Glil reduces the adhesion, migration and invasion of SK-Hepl cells. Our previous findings demonstrated that the aberrant activation of Glil is correlated with invasion and metastasis in HCC tissues (28). Therefore, the effects of Gli1-knockdown on the adhesive, migratory and invasive abilities of hepatoma cells in vitro were determined. First, a cell adhesion assay was performed to test the adhesive ability of the SK-Hep1 cells. The results demonstrated that the adhesive ability was decreased in both $50-$ and $100 \mathrm{nmol} / 1$ si-Gli1 groups compared with the CTRL group $(\mathrm{P}<0.05$; Fig. 3A). The wound healing assay demonstrated that the wound healing rates were markedly inhibited when Gli1 was 
knocked down in hepatoma cells compared with the CTRL group $(\mathrm{P}<0.05$ and $\mathrm{P}<0.01$; Fig. $3 \mathrm{~B}$ and $\mathrm{D})$, suggesting that downregulation of Gli1 expression may suppress the motility of SK-Hep1 cells. Finally, the results of the invasion assay revealed that the invasive capability of the cells was also significantly inhibited after Gli1 was knocked down $(\mathrm{P}<0.01$ and $\mathrm{P}<0.001$; Fig. $3 \mathrm{C}$ and $\mathrm{E}$ ). In addition, NC siRNA had no significant effects on the cell adhesion, migration and invasion of SK-Hep1 cells.

Knockdown of Glil decreases the expression levels of MMP-2 and MMP-9. Accumulating evidence has elucidated that MMPs serve a critical role in the invasion and metastasis of HCC by degrading the extracellular matrix (ECM) components $(29,30)$. Therefore, to determine if Glil silencing-mediated inhibition of cell migration and invasion was associated with MMP-2 and MMP-9, RT-qPCR and western blot analyses was performed to detect their expression. The results revealed that the mRNA and protein expression levels of MMP-2 and MMP-9 were significantly decreased in both si-Glil groups compared with the CTRL group $(\mathrm{P}<0.05, \mathrm{P}<0.01$ and $\mathrm{P}<0.001$; Fig. 4), indicating that inhibition of the migration and invasion of hepatoma cells by Glil depletion may be associated with the downregulation of MMP-2 and MMP-9.

Silencing Glil inhibits FAK/AKT signaling. To explore the crosstalk between Gli1 and the FAK/AKT pathway, FAK, p-FAK, PI3K, p-PI3K, AKT and p-AKT protein expression was detected in SK-Hep1 cells following Gli1-knockdown. The western blotting results demonstrated that the protein levels of p-FAK, p-PI3K and p-AKT were markedly decreased in both si-Glil groups compared with the CTRL group, whereas those of FAK, PI3K and AKT were not significantly different from the CTRL group $(\mathrm{P}<0.05, \mathrm{P}<0.01$ and $\mathrm{P}<0.001$; Fig. $5 \mathrm{~A}$ and $\mathrm{B})$.

The results of immunofluorescence staining were in agreement and demonstrated that the fluorescence intensity of p-FAK localized in the cell membrane was significantly weaker compared with the CTRL group $(\mathrm{P}<0.05$; Fig. $5 \mathrm{C}$ and $\mathrm{E})$ and that of p-AKT located in the nucleus was also significantly weaker in both si-Gli1 groups compared with the CTRL group $(\mathrm{P}<0.01$; Fig. 5D and F). Notably, the intensity of total FAK localized in the cell membrane did not reveal any significant change ( $P>0.05$; Fig. 6 ), which was consistent with the results of western blotting above, suggesting that the observed decrease in p-FAK protein level was not due to a decrease in the amount of total FAK protein.

\section{Discussion}

The results of the present study demonstrated that the efficiency of Gli1 transfected with siRNA was feasible and was independent of its cellular cytotoxicity. Loss of function experiments were performed to elucidate the effects of Gli1 on the biological functions of hepatoma cells. By performing cell adhesion, wound healing and invasion assays after knockdown of Gli1, the data revealed that knockdown significantly reduced the motility and invasion of hepatoma cells. These results indicated that Gli1 serves a crucial role in cell migration and invasion, and provides a potential therapeutic target for HCC.
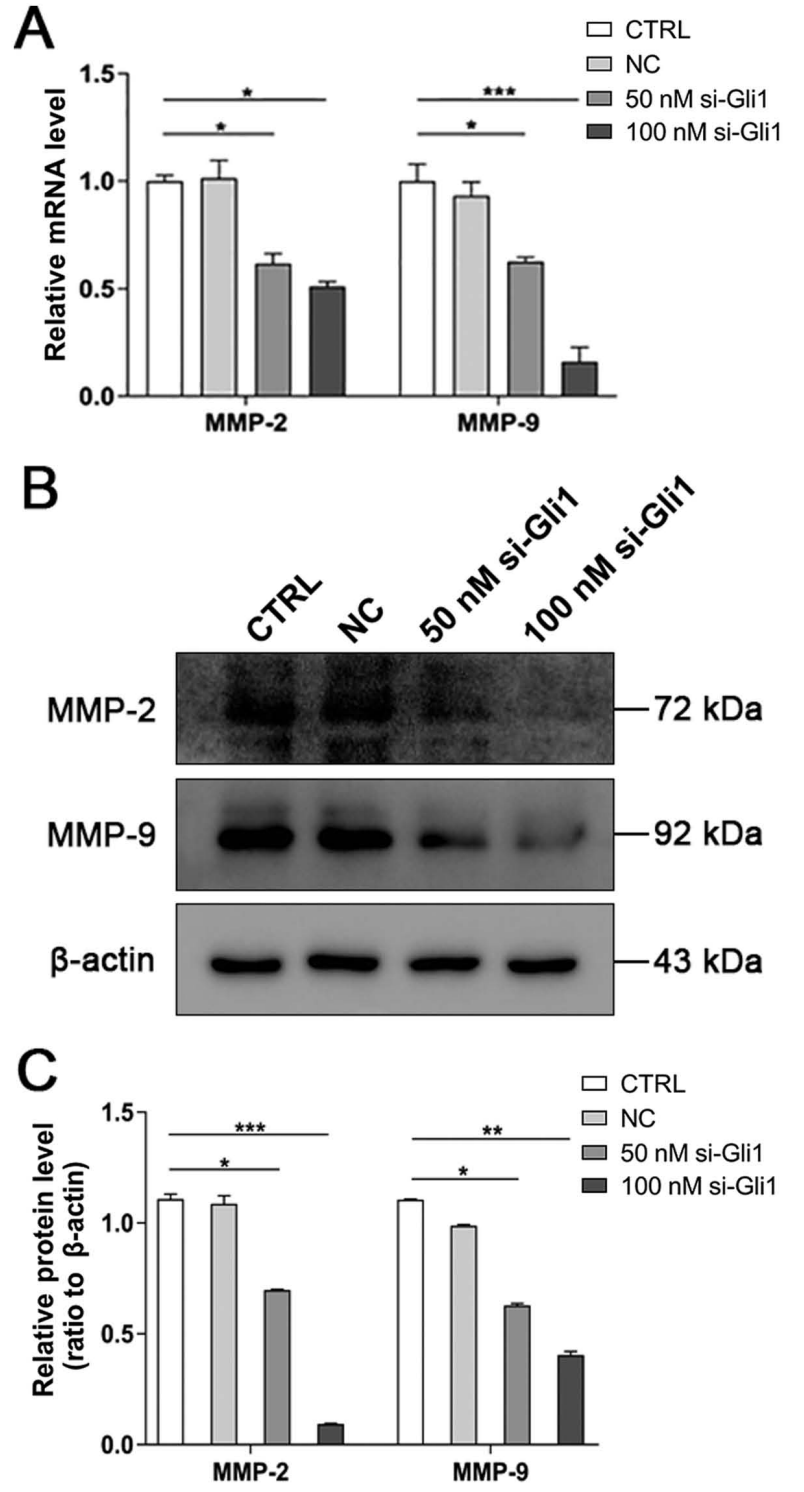

Figure 4. Knockdown of Gli1 decreases the expression of MMP-2 and MMP-9. (A) The relative mRNA expression of MMP-2 and MMP-9 was inhibited in SK-Hep1 cells as demonstrated by reverse transcription-quantitative PCR. (B) The protein expression of MMP-2 and MMP-9 was also inhibited in SK-Hep1 cells, as determined by western blotting. (C) Quantification analysis of representative western blot images using ImageJ software. $\beta$-actin served as a loading control. ${ }^{*} \mathrm{P}<0.05,{ }^{* *} \mathrm{P}<0.01$ and ${ }^{* * *} \mathrm{P}<0.001$ vs. CTRL group. Gill, glioma-associated oncogene homolog 1; si, small interference; CTRL, blank control group; NC, negative control siRNA group.

Cancer invasion and metastasis are complicated multi-step processes in which tumor cells separate from the original site, intravasate into blood vessels, extravasate to a systemic location and colonize at a secondary site (31). Degradation of ECM and proteolysis of the basement membrane are early events in cancer invasion and metastasis $(32,33)$. It has been found that MMPs contain three domains: A catalytic domain, pro-peptide domain and hemagglutinin-like C-terminal domain (34). Tumor cells undergo invasion and metastasis during the early stages of cancer based on their ability to degrade ECM components (35). Among MMPs, MMP-2 and MMP-9 are the two most important factors that degrade type IV collagen, which is the main component of the basement membrane, and serve an essential role in tumor invasion 
A

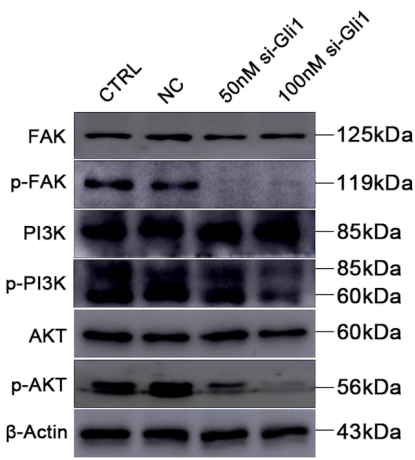

C

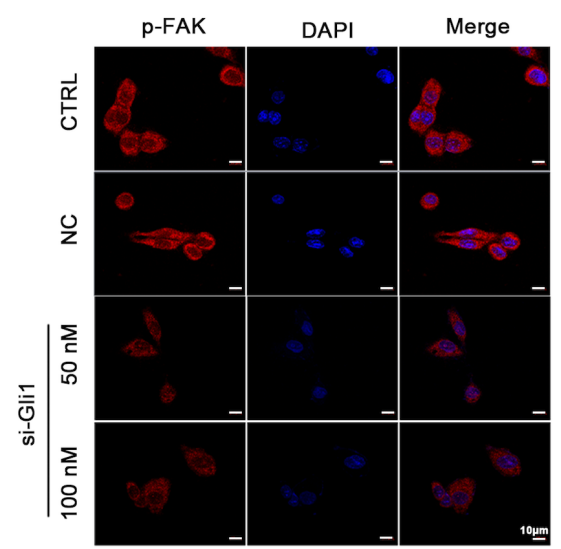

E

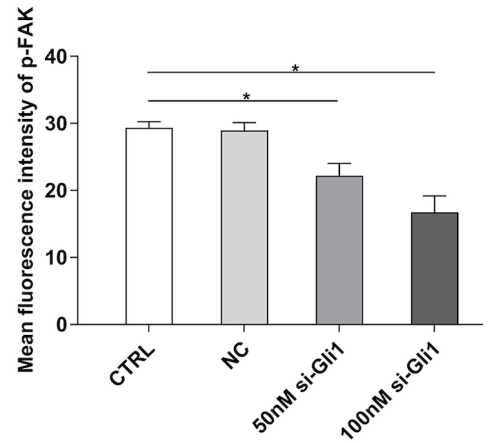

B

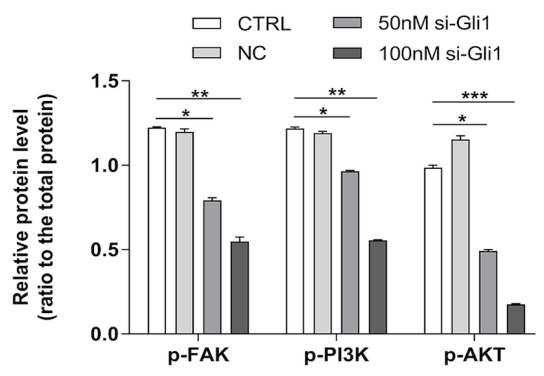

D

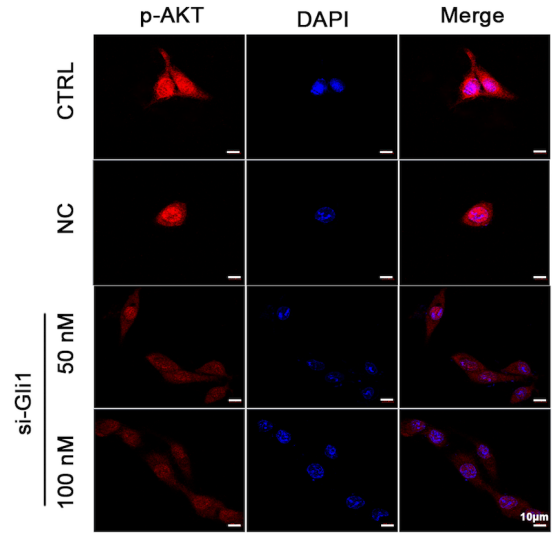

$\mathrm{F}$

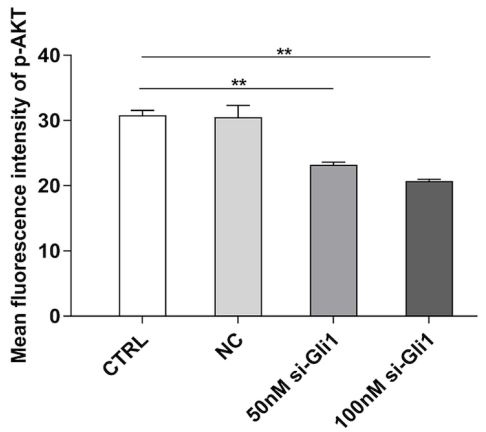

Figure 5. Downregulation of Glil blocks the FAK/AKT signaling pathway. (A) The protein expression levels of FAK, p-FAK, PI3K, p-PI3K, AKT, and p-AKT in SK-Hep1 cells were determined by western blotting. (B) Quantification analysis of representative western blot images using ImageJ software. The phosphorylated epitopes were normalized to the total protein expression, respectively. $\beta$-actin served as a loading control. Representative images of (C) p-FAK and (D) p-AKT immunofluorescence staining in hepatoma SK-Hepl cells. Nuclei were stained with DAPI. Scale bar, $10 \mu \mathrm{m}$. Semi-quantitative analysis of (E) p-FAK and (F) p-AKT fluorescence intensity using ImageJ software. ${ }^{*} \mathrm{P}<0.05,{ }^{* * *} \mathrm{P}<0.01$ and ${ }^{* * * *} \mathrm{P}<0.001$ vs. CTRL group. Gill, glioma-associated oncogene homolog 1; p-, phosphorylated; si, small interference; CTRL, blank control group; NC, negative control siRNA group.

and metastasis (36). Chen et al (15) found that overexpression of MMP-2 and MMP-9 is observed in HCC tissues and is associated with the invasion and metastasis of malignant hepatoma. The present study found that silencing of Gli1 markedly inhibited the mRNA and protein expression of MMP-2 and MMP-9, suggesting that low expression of Gli1 may suppress HCC migration and invasion by regulating the expression of MMP-2 and MMP-9.

A previous study demonstrated that blockade of the FAK/AKT signaling pathway inhibits head and neck cancer metastasis (37). Another study demonstrated that activating the FAK/AKT pathway promotes the epithelial-mesenchymal transition and invasion of HCC (38). Consequently, identifying the crosstalk between Gli1 and FAK/AKT pathway may provide a further understanding of the molecular mechanism of HCC invasion and metastasis. In the present study, Gli1 interference decreased the expression of p-FAK, p-PI3K and p-AKT as known as the active ingredients of the FAK/AKT signaling, while total FAK, PI3K and AKT protein expression was unaltered in the HCC cells. The 
A

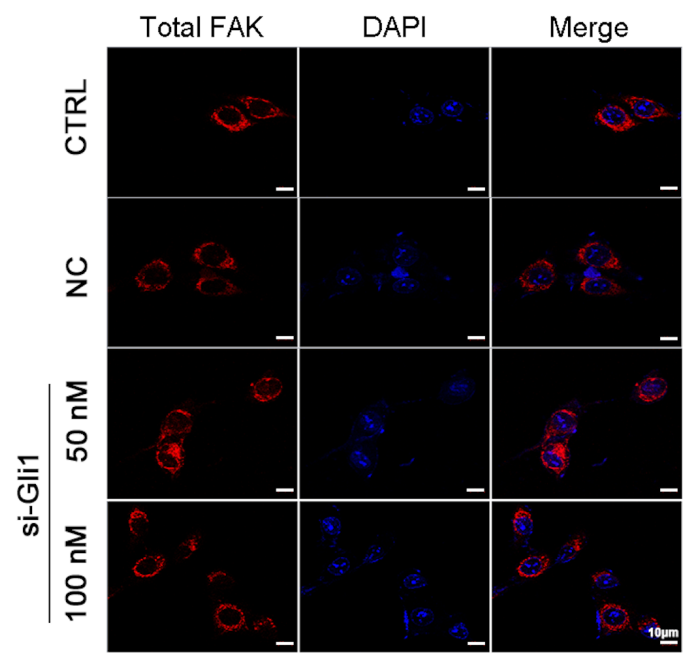

B

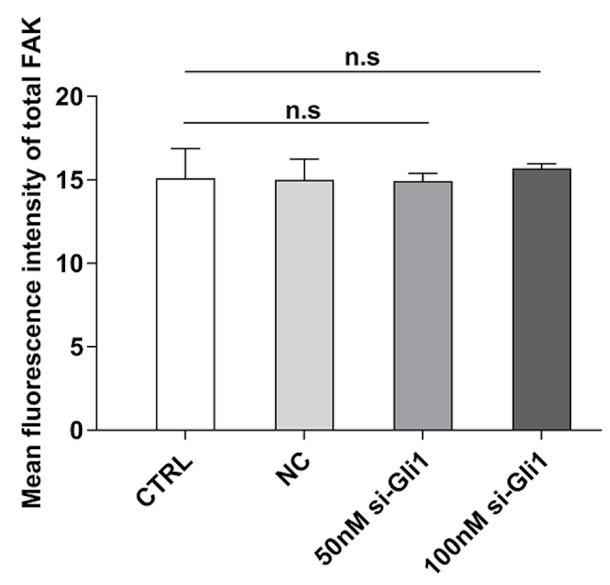

Figure 6. Silencing of Glil does not significantly affect levels of total FAK. (A) Immunofluorescence staining of total FAK in hepatoma SK-Hep1 cells. Nuclei were stained with DAPI. Scale bar, $10 \mu \mathrm{m}$. (B) Semi-quantitative analysis of total FAK fluorescence intensity using ImageJ software. Gil1, glioma-associated oncogene homolog 1; si, small interference; n.s, no significant difference; CTRL, blank control group; NC, negative control siRNA group.

data from the present study indicated that siRNA-mediated depletion of Glil may significantly inhibit the FAK/AKT signaling pathway in vitro. In addition, a previous study demonstrated that phosphorylation of FAK promotes the invasion and metastasis of HCC by upregulating the expression of MMP-2 and MMP-9 (20). Abnormal activation of the PI3K/AKT signaling pathway can promote the proliferation and invasion of tumor cells by regulating the expression of MMP-2 and MMP-9 (39-41). Thus, it was hypothesized that the downregulation of Glil suppressed the invasion and metastasis of HCC cells, which may be achieved by blocking the expression of MMP-2 and MMP-9 mediated through the FAK/AKT signaling pathway.

The present study is not without limitations. First, the present study only assessed the effects of Glil knockdown on the biological functions of hepatoma cells. Prospective studies will upregulate the Gli1 gene to determine the effects of Gli1 on the migratory and invasive abilities of liver cancer cells.
Secondly, the molecular mechanism in the present study is not fully elucidated. Whether downregulation of Gli1 blocks the FAK/AKT signaling pathway, directly or indirectly, requires further investigation via co-immunoprecipitation. Furthermore, understanding protein interactions between the Gli1 and FAK/AKT pathway components may help determine the underlying molecular mechanism presented here.

In conclusion, the results of the present study demonstrated that abnormal Gli1 expression resulted in the significant repression of hepatoma cell migration and invasion via downregulating MMP-2 and MMP-9 by blocking the FAK/AKT signaling pathway. These observations revealed novel biological insights into the mechanisms underlying HCC invasion and metastasis, and highlight Glil as a potential therapeutic target for HCC.

\section{Acknowledgements}

Not applicable.

\section{Funding}

The present study was funded by grants from the National Natural Science Foundation of China (grant no. 81760160), the Natural Science Foundation of Jiangxi Province (grant no. 20151BAB205044), the Science and Technology Research Project of Jiangxi Provincial Education Department (grant no. 180797), the Provincial Innovation Training Project of Jiangxi Provincial Undergraduates (grant no. S202010413009) and the Science and Technology Innovation Project for Undergraduates of Gannan Medical University (grant no. BKSZR201902).

\section{Availability of data and materials}

All data generated or analyzed during the present study are available from the corresponding authors upon reasonable request.

\section{Authors' contributions}

JNZ and BC conceived and designed the study. ZH, FX, AH and MX performed the experiments and collected the data. YL and JKZ analyzed the data and ZH wrote the manuscript. JX and YS replicated the experiments and revised the figures. JNZ and BC critically revised the manuscript for important intellectual content. All authors read and approved the final manuscript.

\section{Ethics approval and consent to participate}

Not applicable.

\section{Patient consent for publication}

Not applicable.

\section{Competing interests}

The authors declare that they have no competing interests. 


\section{References}

1. Bray F, Ferlay J, Soerjomataram I, Siegel RL, Torre LA and Jemal A: Global cancer statistics 2018: GLOBOCAN estimates of incidence and mortality worldwide for 36 cancers in 185 countries. CA Cancer J Clin 68: 394-424, 2018.

2. Torre LA, Bray F, Siegel RL, Ferlay J, Lortet-Tieulent J and Jemal A: Global cancer statistics, 2012. CA Cancer J Clin 65: 87-108, 2015

3. Mikhail S, Cosgrove D and Zeidan A: Hepatocellular carcinoma: Systemic therapies and future perspectives. Expert Rev Anticancer Ther 14: 1205-1218, 2014.

4. Baek KK, Kim JH, Uhm JE, Park SH, Lee J, Park JO, Park YS, Kang WK and Lim HY: Prognostic factors in patients with advanced hepatocellular carcinoma treated with sorafenib: A retrospective comparison with previously known prognostic models. Oncology 80: 167-174, 2011.

5. Ikeda M, Morizane C, Ueno M, Okusaka T, Ishii H and Furuse J: Chemotherapy for hepatocellular carcinoma: Current status and future perspectives. Jpn J Clin Oncol 48: 103-114, 2018.

6. Skoda AM, Simovic D, Karin V, Kardum V, Vranic S and Serman L: The role of the Hedgehog signaling pathway in cancer: A comprehensive review. Bosn J Basic Med Sci 18: 8-20, 2018.

7. Wang Y, Han C, Lu L, Magliato S and Wu T: Hedgehog signaling pathway regulates autophagy in human hepatocellular carcinoma cells. Hepatology 58: 995-1010, 2013.

8. Ma Y, Yu W, Shrivastava A, Alemi F, Lankachandra K, Srivastava RK and Shankar S: Sanguinarine inhibits pancreatic cancer stem cell characteristics by inducing oxidative stress and suppressing sonic hedgehog-Gli-Nanog pathway. Carcinogenesis 38: 1047-1056, 2017.

9. Magistri P, Leonard SY, Tang CM, Chan JC, Lee TE and Sicklick JK: The glypican 3 hepatocellular carcinoma marker regulates human hepatic stellate cells via Hedgehog signaling. J Surg Res 187: 377-385, 2014.

10. Ke Z, Caiping S, Qing Z and Xiaojing W: Sonic hedgehog-Gli1 signals promote epithelial-mesenchymal transition in ovarian cancer by mediating PI3K/AKT pathway. Med Oncol 32: 368, 2015.

11. Sanchez P, Hernández AM, Stecca B, Kahler AJ, DeGueme AM, Barrett A, Beyna M, Datta MW, Datta S and Ruiz i Altaba A Inhibition of prostate cancer proliferation by interference with SONIC HEDGEHOG-GLI1 signaling. Proc Natl Acad Sci USA 101: 12561-12566, 2004.

12. Jian-Hui C, Er-Tao Z, Si-Le C, Hui W, Kai-Ming W, Xin-Hua Z, Chuang-Qi C, Shi-Rong C and Yu-Long H: CD44, sonic hedgehog, and Gli1 expression are prognostic biomarkers in gastric cancer patients after radical resection. Gastroenterol Res Pract 2016: 1013045, 2016.

13. Min S, Xiaoyan X, Fanghui P, Yamei W, Xiaoli Y and Feng W: The glioma-associated oncogene homolog 1 promotes epithelial-mesenchymal transition in human esophageal squamous cell cancer by inhibiting E-cadherin via Snail. Cancer Gene Ther 20 379-385, 2013

14. Sheng W, Dong M, Zhou J, Li X, Liu Q, Dong Q and Li F: The clinicopathological significance and relationship of Gli1, MDM2 and p53 expression in resectable pancreatic cancer. Histopathology 64: 523-535, 2014.

15. Chen JS, Huang XH, Wang Q, Huang JQ, Zhang LJ, Chen XL, Lei J and Cheng ZX: Sonic hedgehog signaling pathway induces cell migration and invasion through focal adhesion kinase/AKT signaling-mediated activation of matrix metalloproteinase (MMP)-2 and MMP-9 in liver cancer. Carcinogenesis 34: 10-19, 2013.

16. Liu CH, Lan CT, Chou JF, Tseng TJ and Liao WC: CHSY1 promotes aggressive phenotypes of hepatocellular carcinoma cells via activation of the hedgehog signaling pathway. Cancer Lett 403: 280-288, 2017.

17. Guo D, Zhang D, Ren M, Lu G, Zhang X, He S and Li Y: THBS4 promotes HCC progression by regulating ITGB1 via FAK/PI3K/AKT pathway. FASEB J, Jun 22, 2020 (Epub ahead of print).

18. Tokunaga E, Oki E, Egashira A, Sadanaga N, Morita M, Kakeji Y and Maehara Y: Deregulation of the Akt pathway in human cancer. Curr Cancer Drug Targets 8: 27-36, 2008.

19. Chen HC, Appeddu PA, Isoda H and Guan JL: Phosphorylation of tyrosine 397 in focal adhesion kinase is required for binding phosphatidylinositol 3-kinase. J Biol Chem 271: 26329-26334, 1996.

20. Chen JS, Huang XH, Wang Q, Chen XL, Fu XH, Tan HX, Zhang LJ, $\mathrm{Li} \mathrm{W}$ and $\mathrm{Bi}$ J: FAK is involved in invasion and metastasis of hepatocellular carcinoma. Clin Exp Metastasis 27: 71-82, 2010.
21. Cance WG, Harris JE, Iacocca MV, Roche E, Yang X, Chang J, Simkins S and Xu L: Immunohistochemical analyses of focal adhesion kinase expression in benign and malignant human breast and colon tissues: Correlation with preinvasive and invasive phenotypes. Clin Cancer Res 6: 2417-2423, 2000.

22. Owens LV, Xu L, Craven RJ, Dent GA, Weiner TM, Kornberg L, Liu ET and Cance WG: Overexpression of the focal adhesion kinase (p125FAK) in invasive human tumors. Cancer Res 55: 2752-2755, 1995.

23. Owens LV, Xu L, Dent GA, Yang X, Sturge GC, Craven RJ and Cance WG: Focal adhesion kinase as a marker of invasive potential in differentiated human thyroid cancer. Ann Surg Oncol 3: 100-105, 1996.

24. Tremblay L, Hauck W, Aprikian AG, Begin LR, Chapdelaine A and Chevalier S: Focal adhesion kinase (pp125FAK) expression, activation and association with paxillin and p50CSK in human metastatic prostate carcinoma. Int J Cancer 68: 164-171, 1996.

25. Ayaki M, Komatsu K, Mukai M, Murata K, Kameyama M, Ishiguro S, Miyoshi J, Tatsuta $\mathrm{M}$ and Nakamura H: Reduced expression of focal adhesion kinase in liver metastases compared with matched primary human colorectal adenocarcinomas. Clin Cancer Res 7: 3106-3112, 2001.

26. Xia H, Nho RS, Kahm J, Kleidon J and Henke CA: Focal adhesion kinase is upstream of phosphatidylinositol 3-kinase/Akt in regulating fibroblast survival in response to contraction of type I collagen matrices via a beta 1 integrin viability signaling pathway. J Biol Chem 279: 33024-33034, 2004

27. Pfaffl MW: A new mathematical model for relative quantification in real-time RT-PCR. Nucleic Acids Res 29: e45, 2001.

28. Chen B, Hu Z, Li B, Lin X, Luo Z and Hu Z: The expressions of Hedgehog and PI3K-AKT pathway components correlate with invasion and metastasis in hepatocellular carcinoma. Int J Clin Exp Pathol 12: 2381-2388, 2019.

29. Yamamoto H, Itoh F, Adachi Y, Sakamoto H, Adachi M, Hinoda Y and Imai K: Relation of enhanced secretion of active matrix metalloproteinases with tumor spread in human hepatocellular carcinoma. Gastroenterology 112: 1290-1296, 1997.

30. Kessenbrock K, Plaks V and Werb Z: Matrix metalloproteinases: Regulators of the tumor microenvironment. Cell 141: 52-67, 2010.

31. Chaffer CL and Weinberg RA: A perspective on cancer cell metastasis. Science 331: 1559-1564, 2011.

32. Coussens LM, Fingleton B and Matrisian LM: Matrix metalloproteinase inhibitors and cancer: Trials and tribulations. Science 295: 2387-2392, 2002.

33. Stetler-Stevenson WG: Type IV collagenases in tumor invasion and metastasis. Cancer Metastasis Rev 9: 289-303, 1990.

34. Vihinen P and Kähäri VM: Matrix metalloproteinases in cancer: Prognostic markers and therapeutic targets. Int $\mathbf{J}$ Cancer 99: 157-166, 2002.

35. Hadler-Olsen E, Winberg JO and Uhlin-Hansen L: Matrix metalloproteinases in cancer: Their value as diagnostic and prognostic markers and therapeutic targets. Tumour Biol 34: 2041-2051,2013.

36. Parnham A and Bousfield C: The influence of matrix metalloproteases and biofilm on chronic wound healing: A discussion. Br J Community Nurs 23 (Suppl 3): S22-S29, 2018.

37. Kim SA, Kwon SM, Kim JA, Kang KW, Yoon JH and Ahn SG: 5'-Nitro-indirubinoxime, an indirubin derivative, suppresses metastatic ability of human head and neck cancer cells through the inhibition of Integrin $\beta 1 / F A K / A k t$ signaling. Cancer Lett 306 : 197-204, 2011.

38. Wang R, Yu Z, Chen F, Xu H, Shen S, Chen W, Chen L, Su Q, Zhang L, Bi J, et al: miR-300 regulates the epithelial-mesenchymal transition and invasion of hepatocellular carcinoma by targeting the FAK/PI3K/AKT signaling pathway. Biomed Pharmacother 103: 1632-1642, 2018.

39. Cheng JC, Chou CH, Kuo ML and Hsieh CY: Radiation-enhanced hepatocellular carcinoma cell invasion with MMP-9 expression through PI3K/Akt/NF-kappaB signal transduction pathway. Oncogene 25: 7009-7018, 2006.

40. Shukla S, Maclennan GT, Hartman DJ, Fu P, Resnick MI and Gupta S: Activation of PI3K-Akt signaling pathway promotes prostate cancer cell invasion. Int J Cancer 121: 1424-1432, 2007.

41. Li H, Zhang Y, Hai J, Wang J, Zhao B, Du L and Geng X: Knockdown of TRIM31 suppresses proliferation and invasion of gallbladder cancer cells by down-regulating MMP2/9 through the PI3K/Akt signaling pathway. Biomed Pharmacother 103: 1272-1278, 2018.

This work is licensed under a Creative Commons Attribution-NonCommercial-NoDerivatives 4.0 International (CC BY-NC-ND 4.0) License. 